\title{
Meningitis por Cryptococcus neoformans en niños y adolescentes infectados por virus de la inmunodeficiencia humana
}

\author{
Jorge Quian, Stella Gutiérrez, Virginia González, Mercedes Sánchez, Marina Abayian y Fernando Baccino
}

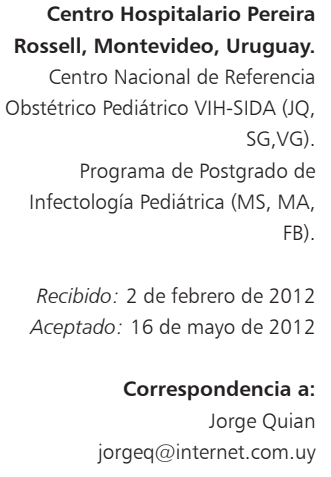

Centro Hospitalario Pereira Rossell, Montevideo, Uruguay Centro Nacional de Referencia SG,VG)

\section{Cryptococcus neoformans meningitis in children and adolescentes infected with HIV}

Three cases of meningitis due to criptococcus in children infected with HIV are reported. All patients had severe immunodeficiency. The first case occurred before antiretroviral therapy. The second case had poor adherence to treatment. The third case is a teenager with prolonged fever, who did not have a clear source of transmission of HIV and was immunodeficient at the time of diagnosis. Examination of cerebrospinal fluid with India ink dye was essential for making the diagnosis in all three cases. The disease course was adequate in all three patients despite flucytosine not being available.

Key words: HIV, Immunodeficiency, Cryptococcus, meningitis.

Palabras clave: VIH, Inmunodeficiencia, Cryptococcus, meningitis.

\section{Introducción}

L a evolución de la infección por virus de inmunodeficiencia humana (VIH) luego de la introducción del tratamiento anti-retroviral de alta efectividad (TAR) ha sido extraordinaria. De ser una enfermedad con pronóstico fatal a más o menos largo plazo, se ha transformado en una enfermedad crónica ${ }^{1-3}$. Entre las mejoras obtenidas, las infecciones por microorganismos oportunistas se han reducido de manera significativa ${ }^{4-6}$.

La infección por Cryptococcus sp., sobre todo en su forma clínica de meningitis, era una complicación frecuente entre los pacientes con infección por VIH y se consideraba que 10 a $30 \%$ de quienes no recibían TAR podían desarrollar criptococosis. En un número importante de casos esta enfermedad oportunista define el síndrome de inmunodeficiencia adquirida (SIDA) ${ }^{7-10}$.

En Uruguay, las publicaciones científicas en población adulta muestran que 5,6\% de los pacientes con SIDA tenían el antecedente de criptococosis y posteriormente se comunicó que hasta en 3\% de los pacientes con SIDA fallecidos fue la primera patología marcadora del síndrome ${ }^{11,12}$.

El uso de TAR ha producido una disminución significativa en la incidencia de la criptococosis ${ }^{13-15}$. Se han realizado estimaciones que en el año 2006 se podrían haber producido unos 957.000 casos de meningitis criptocóccica en el mundo y que 624.725 personas podrían haber fallecido por esta causa ${ }^{16}$. En ese año ya estaba a disposición en gran parte del mundo la TAR, pero un porcentaje importante de individuos no la recibía.

La prevalencia de meningitis por Cryptococcus sp. en niños con SIDA es menor que en adultos, se estima que en la población pediátrica sería de $1 \%{ }^{17}$ en tanto que en adultos llegaría a 6-8\%.

Cryptococcus sp. es un hongo encapsulado, considerado un patógeno no habitual del que se conocían pocos casos antes de 1955 y que se transformó en un microorganismo oportunista frecuente en todo el mundo en la medida que aumentó la población de personas inmunocomprometidas ${ }^{18}$. El hongo penetra al organismo por vía respiratoria pero tiene especial predilección por afectar el sistema nervioso central. La meningitis criptocóccica es la principal manifestación clínica asociada a una alta mortalidad.

Hay observaciones que sugieren que existen infecciones por Cryptococcus sp. asintomáticas y frecuentes ya en los primeros años de vida ${ }^{19}$.

Se presentan tres casos de meningitis criptocócoccica en pacientes con infección por VIH en edad pediátrica caracterizadas por inmunodepresión grave.

\section{Casos clínicos}

\section{Caso 1}

Varón de 7 años con infección por VIH adquirida por transmisión vertical, diagnosticada a los 5 años de edad. Consultó por lesiones cutáneas maculares con un centro necrótico (Figura 1). Posteriormente, se agregó episodios de cefaleas intensas asociadas a fiebre. Fue internado con 
diagnóstico probable de meningitis. Dentro del estudio de líquido céfalo-raquídeo (LCR) se realizó tinción con tinta china que resultó positiva y en el cultivo hubo aislamiento de Cryptococcus neoformans. En ese momento, tenía linfocitos CD4 de 158 céls $/ \mathrm{mm}^{3}$ (9\%). Recibió tratamiento con anfotericina B deoxicolato i.v y posteriormente fluconazol por vía oral con buena evolución. Se mantuvo hospitalizado con tratamiento antifúngico durante 42 días. Tres meses después se inició monoterapia con zidovudina (AZT), que luego de 8 meses se cambió a didanosina (ddI). Posteriormente, recibió biterapia con ddI y ritonavir. Falleció a los 8 años de edad.

\section{Caso 2}

Varón de 10 años con infección por VIH por transmisión vertical, diagnosticada a los 2 años de edad. Tenía antecedente de mala adherencia a varios regímenes de tratamiento propuestos, su madre también había rechazado la TAR. Había sido internado en varias ocasiones por enfermedades intercurrentes por microorganismos habituales y oportunistas. Fue internado por una historia de 5 días de dolor abdominal, acompañados de fiebre hasta $38,5^{\circ} \mathrm{C}$ y un exantema máculo-papular generalizado. En el momento del ingreso se constató además del exantema, una desnutrición secundaria, mal estado general, adenopatías cervicales, estertores húmedos en ambos campos pulmonares, caries dentales y otorrea purulenta a izquierda. En ese momento tenía una carga viral (CV) de 95.000 copias $/ \mathrm{ml}$ y linfocitos CD4 de 26 céls $/ \mathrm{mm}^{3}$. Se inició antibioterapia, sin embargo, a las 48 h persistía con fiebre a la que se agregó cefalea intensa, vómitos, fotofobia y acusofobia.

Se realizó un TAC de cráneo que resultó normal; el análisis citoquímico de LCR: glucosa $0,43 \mathrm{~g} / 1$, proteínas 1,15 gr/l, celularidad: 5 leucocitos $/ \mathrm{mm}^{3}$; el examen microbiológico directo (tinta china) y cultivo fueron positivos para C. neoformans. Se trató con anfotericina B deoxicolato i.v. durante 21 días y posteriormente fluconazol por vía oral en plan de mantención. Hubo mejoría clínica y el estudio de LCR a los 21 días fue normal con cultivo negativo. Fue dado de alta a los 33 días y al mes de evolución ya evidenciaba aumento de peso y buen estado general. El test de genotipificación no mostró mutaciones asociadas a resistencia por lo que se inició tratamiento con AZT, lamivudina (3TC) y lopinavir/ritonavir.

\section{Caso 3}

Adolescente femenina de 13 años, sin antecedentes mórbidos. Fue internada por un síndrome febril de un mes de evolución, con lesiones cutáneas no bien catalogadas y una celulitis en el tercio inferior de la pierna izquierda. Presentaba una repercusión nutricional importante con un IMC 14,1 ( $Z<-2)$. Dentro de su estudio se realizó ELISA y Westernblot para VIH que resultaron positivos;

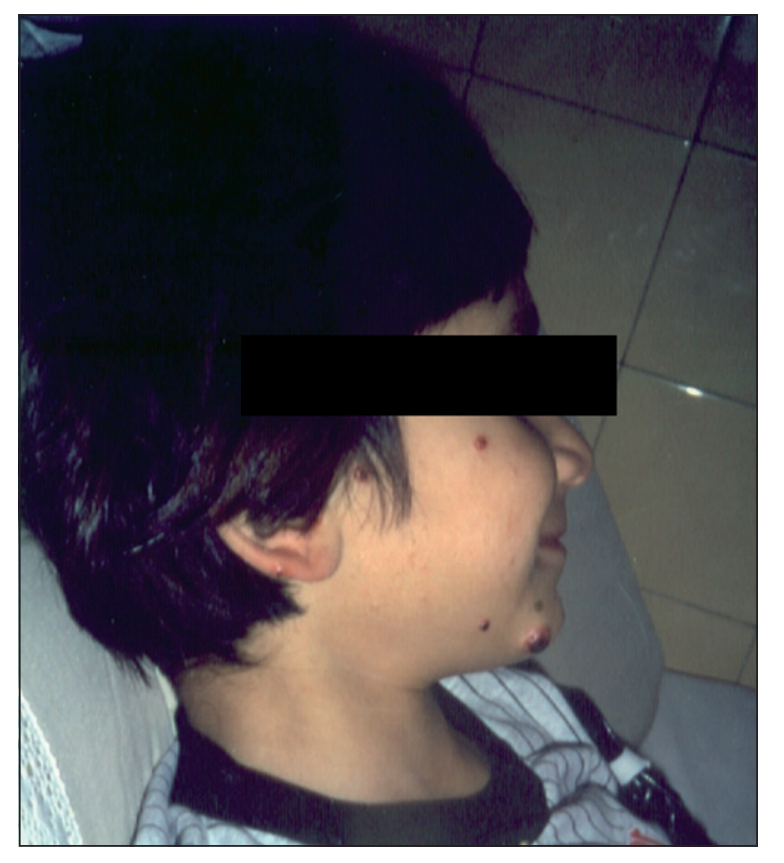

Figura 1.

además se estudió a la madre, el padre y los hermanos que tuvieron serología negativa. No había antecedentes de hospitalizaciones ni transfusiones de productos sanguíneos. Los familiares negaron la posibilidad de abuso sexual y el examen ginecológico fue normal. Evolucionó con mejoría clínica del proceso infeccioso agudo con tratamiento antibacteriano, por lo que se inició tratamiento con anti-retrovirales: AZT, 3TC y lopinavir/ritonavir, con linfocitos CD4 18 céls $/ \mathrm{mm}^{3}$ y CV de 130.000 copias $/ \mathrm{ml}$.

Posteriormente, presentó varios episodios interpretados como reacciones cutáneas a la terapia por lo que fue necesario el uso de antihistamínicos y corticoesteroides. A los dos meses de iniciada la TAR fue internada por fiebre, diplopía, cefalea y vómitos por lo que se diagnosticó una meningitis aguda. El análisis citoquímico de LCR: glucosa $0,39 \mathrm{~g} / 1$, proteínas $0,57 \mathrm{~g} / 1$, lactato $2,03 \mathrm{mmol} / 1$, leucocitos: no se observaron y eritrocitos: $520 / \mathrm{mm}^{3}$. La tinción con tinta china fue positiva y en el cultivo hubo aislamiento de $C$. neoformans. Se trató con anfotericina B deoxicolato i.v. durante 10 días y luego anfotericina liposomal por desarrollar insuficiencia renal secundaria. Se realizó una resonancia magnética que fue normal y un fondo de ojo que mostró un edema de papila bilateral. A los 15 días se realizó control de LCR, análisis citoquímico: glucosa 0,21 g/l, proteínas 1,57 g/l, lactato 3,59 mmol/1, leucocitos: no se observaron, eritrocitos: $200 / \mathrm{mm}^{3}$ conservados. El cultivo de LCR persistió positivo para $C$. neoformans, negativizándose al mes de tratamiento; sin embargo, presentó intensificación de la cefalea y convulsiones debiendo trasladarse a unidad de terapia intensiva. Su deterioro clínico fue interpretado como un síndrome de 
respuesta inflamatoria inmune, por lo que fue manejada con corticoesteroides por vía i.v. Evolucionó en buenas condiciones, siendo dado de alta a su domicilio luego de 51 días de hospitalización. La paciente mantuvo TAR con aumento progresivo de linfocitos CD4 hasta $240 / \mathrm{mm}^{3}$ y $\mathrm{CV}$ indetectables.

\section{Discusión}

La gran mayoría de las pacientes con criptococosis sintomática diseminada tienen una condición subyacente de inmunocompromiso, siendo el SIDA la más frecuente. La tasa de criptococosis en E.U.A en la era pre-SIDA era aproximadamente de 0,8 casos por un millón de personas por año. En 1992, durante el pico de la epidemia, la tasa alcanzó casi 5 casos por cien mil personas por año. Con el inicio de la TAR y el uso de fluconazol para el tratamiento de la candidiasis oral, la tasa se redujo y se estabilizó en aproximadamente 1 caso por 100.000 personas por año ${ }^{20,21}$.

La infección por Cryptococcus sp. se presenta con menor frecuencia en niños que en adultos. En E.U.A. la incidencia de la enfermedad en niños con SIDA, parece ser de alrededor de 0,5 a $1 \%{ }^{17,22}$, aunque se han reportado cifras mayores en Sudáfrica y Tailandia ${ }^{23}$.

En el Centro Nacional de Referencia Obstétrico Pediátrico en Uruguay, desde junio de 1990 hasta junio de 2011, se han asistido 221 niños infectados con VIH y tres han sido diagnosticados con meningitis criptocóccica, lo que significa $1,3 \%$.

Los tres casos clínicos relatados representan las distintas formas en que se presentan los niños con criptococosis; tienen en común la profunda inmunodepresión y no recibir TAR por distintas circunstancias ${ }^{10}$.

El primer caso ocurrió en un niño portador de VIH en la época en que en Uruguay no se contaba con TAR. Como se observa en la evolución cronológica, cuando se inició la TAR primero fue con monoterapia y posteriormente con biterapia, posterior a la meningitis por Cryptococcus sp. La afección cutánea era llamativa, pero de ninguna manera típica. La piel, luego de la infección pulmonar y del sistema nervioso central es el blanco principal de la enfermedad y las lesiones se presentan bajo muy diferentes aspectos ${ }^{24,25}$. Los tres pacientes tuvieron compromiso de la piel de distintas formas.

La evolución a la muerte luego de la criptococosis, como en el primer paciente, era habitual en quienes no recibían $\mathrm{TAR}^{26,27}$.

El segundo caso fue un niño no adherente a la terapia, con una inmunodepresión grave. Su falta de adherencia se reafirmó además por un test de genotipificación sin evidenciar mutaciones asociadas a resistencia.
Por último en la adolescente, en quien no fue posible encontrar la fuente de transmisión, se hizo el diagnóstico de infección por VIH con una inmunosupresión avanzada. En la población con SIDA de los países desarrollados, la criptococosis representa en la actualidad una infección que identifica a un paciente con mal pronóstico o una infección por VIH sin diagnosticar y sin tratar; estos casos son ejemplos de ello.

Los síntomas predominantes en los tres fueron la fiebre y la cefalea; frente a ellos en un paciente inmunocomprometido debe pensarse en criptococosis y luego de obtener LCR realizar examen con tinta china que rápidamente permite confirmar el diagnóstico. Aproximadamente $80 \%$ de los pacientes con SIDA y $50 \%$ de los que no tienen SIDA se observa el hongo en la tinción con tinta china ${ }^{18}$.

Es necesario controlar muestras de LCR para certificar la erradicación de Cryptococcus sp. Un cultivo estéril a las dos semanas de iniciado el tratamiento permite confirmar la acción fungicida de los fármacos y esto se asocia con éxito terapéutico ${ }^{28}$.

El tratamiento ideal es anfotericina B asociado a flucitosina que no fue posible realizarlo en ningún paciente ya que esta último fármaco no estaba disponible en nuestro país $^{29,30}$. En todos se hizo terapia de mantenimiento con fluconazol, ya que sin esta medida de profilaxis secundaria se observa una alta tasa de recaídas ${ }^{28,31}$.

En suma, se quiere compartir la experiencia con tres pacientes inmunocomprometidos, en este caso infectados por $\mathrm{VIH}$, que desarrollaron cuadros caracterizados fundamentalmente por fiebre y cefalea. El conocimiento de que se trata de un niño con inmunidad alterada, debe hacer plantear al médico clínico esta entidad y solicitar tinción con tinta china del LCR que puede rápidamente permitir el diagnóstico y tratamiento adecuado.

\section{Resumen}

Se presentan tres casos de meningitis por Cryptococcus sp. en niños infectados con VIH e inmunodeficiencia grave. El primero fue en la época en que no se disponía de TAR. El segundo fue un paciente no adherente al tratamiento. La tercera fue una adolescente en la que se hizo diagnóstico por una enfermedad febril prolongada, donde no fue posible encontrar la fuente de transmisión de VIH y que en el momento del diagnóstico estaba con inmunosupresión grave. En los tres fue fundamental para el diagnóstico rápido la tinción de LCR con tinta china. La evolución de la enfermedad fue adecuada en los tres pacientes aunque no se contó en ninguno de los casos con flucitosina. 


\section{Referencias bibliográficas}

1.- Gortmaker S L, Hughes M, Cervia J, Brady M, Johnson G M, Seage G R, et al. Effect of combination therapy including protease inhibitors on mortality among children and adolescents with HIV-1. N Engl J Med 2001; 345: 1522-8.

2.- Gona P, Van Dyke R B, Williams P L, Dankner W M, Chernoff M C, Nachman S A, et al. Incidence of opportunistic infections and other infections in HIV-infected children in the HAART era. JAMA 2006; 296: 292-300.

3.- Gibb D M, Duong T, Tookey P A, Sharland M, Tudor-Williams G, Novelli V, et al. Decline in mortality, AIDS and hospital admissions in perinatally HIV-infected children in the United Kingdom and Ireland. BMJ 2003; 327: 1019.

4.- Nesheim S R, Kapogiannis B G, Soe M M, Sullivan K M, Abrams E, Farley J, et al. Trends in opportunistic infections in the pre-and posthighly active antiretroviral therapy eras among HIV-infected children in the Perinatal AIDS Collaborative Transmission Study, 1986-2004. Pediatrics 2007; 120: 100-9.

5.- Ylitalo N, Brogly S, Hughes M D, Nachman S, Dankner W, Van Dyke R, et al. Risk factors for opportunistic illnesses in children with HIV in the era of highly active antiretroviral therapy. Arch Pediatr Adolesc Med 2006; 160 : 778-87.

6.- Mirza S A, Phelan M, Rimland D, Graviss E, Hamill R, Brandt M E, et al. The changing epidemiology of cryptococcosis: an update from population-based active surveillance in 2 large metropolitan areas, 1992-2000. Clin Infect Dis 2003; 36: 789-94.

7.- Dromer F, Mathoulin S, Dupont B, Laporte A. Epidemiology of cryptococcosis in France: a 9-year survey (1985-1993). French Cryptococcosis Study Group. Clin Infect Dis 1996; 23: 82-90.

8.- Currie B P, Casadevall A. Estimation of the prevalence of cryptococcal infection among patients infected with the HIV in New York City. Clin Infect Dis 1994; 19: 1029-33.

9.- Hakim J G, Gangaidzo I T, Heyderman R S, Mielke J, Mushangi E, Taziwa A, et al. Impact of HIV infection on meningitis in Harare, Zimbabwe: a prospective study of 406 predominantly adults patients. AIDS 2000; 14 : 1401-7.
10.- Lee S J, Choi H K, Son J, Kim K H, Lee S H Cryptococcal meningitis in patients with or without human immunodeficiency virus: experience in a tertiary hospital. Yonsei Med J 2011; 52: 482-7.

11.- Mansilla M, Braselli A, Purtscher H, Dutra A, Oliver L, Gezuele E. Criptococosis en pacientes con síndrome de inmunodeficiencia adquirida (SIDA). Rev Med Urug 1992; 8: 104-7.

12.- Mansilla M, Purtscher H, Mirazo I, Lima E, Cardozo A, Braselli A. Evolución de 172 pacientes VIH (+) fallecidos en Uruguay. Rev Med Urug 1996; 12: 123-34.

13.- Dromer F, Mathoulin-Pélissier S, Fontanet A, Ronin O, Dupont B, Lortholary O. Epidemiology of HIV-associated cryptococcosis in France (1985-2001): comparison of the preand post-HAART eras. AIDS 2004, 18: 555-62.

14.- d'Arminio-Montforte A, Duca P G, Vago L, Grassi M P, Moroni M. Decreasing incidence of CNS AIDS-defining events associated with antiretroviral therapy. Neurology 2000; 54 : 1856-9.

15.- Kaplan J E, Hanson D, Dworkin M S, Frederick T, Bertolli J, Lindegren M L, et al. Epidemiology of human immunodeficiency virus-associated opportunistic infections in the United States in the era of highly active antiretroviral therapy. Clin Infect Dis 2000; 30 (Suppl 1): S5-14.

16.- Park B J, Wannemuehler K A, Marston B J, Govender N, Pappas P G, Chiller T M. Estimation of the current global burden of cryptococcal meningitis among persons living with HIV/AIDS. AIDS 2009; 23: 525-30.

17.- Abadi J, Nachman S, Kressel A B, Pirofski L. Cryptococcosis in children with AIDS. Clin Infect Dis 1999; 28: 309-13.

18.- Perfect J R, Casadevall A. Cryptococcosis. Infect Dis Clin North Am 2002; 16: 837-74.

19.- Goldman D L, Khine H, Abadi J, Lindenberg D, Pirofski L, Niang R, et al. Serologic evidence for Cryptococcus neoformans infection in early childhood. Pediatrics 2001; 107: e66.

20.- Hajjeh R, Conn L A, Stephens D S, Baughman W, Hamill R, Graviss E, et al. Cryptococcosis: population-based multistate active surveillance and risk factors in human immunodeficiency virus-infected persons. Cryptococcal Active Surveillance Group. J Infect Dis 1999; 179: 449-54.

21.- van Elden L J, Walenkamp A M, Lipovsky M
M, Reiss P, Meis J F, de Marie S, et al. Declining number of patients with cryptococcosis in the Netherlands in the era of highly active antiretroviral therapy. AIDS 2000; 14: 2787-8.

22.- González C E, Shetty D, Lewis L L, Mueller B U, Pizzo P A, Walsh T J. Cryptococcosis in human immunodeficiency virus-infected children. Pediatr Infect Dis J 1996; 15: 796-800.

23.- McCarthy K M, Morgan J, Wannemuehler K A, Mirza S A, Gould S M, Mhlongo N, et al. Population-based surveillance for cryptococcosis in an antiretroviral-naive South African province with a high HIV seroprevalence. AIDS 2006; 20: 2199-206.

24.- Concus A P, Helfand R F, Imber M J, Lerner E A, Sharpe R J. Cutaneous cryptococcosis mimicking molluscum contagiosum in a patient with AIDS. J Infect Dis 1988; 158: 897-8.

25.- Gauder J P. Cryptococcal cellulites. JAMA 1977; 237: 672-3.

26.- Powderly W G. Cryptococcal meningitis and AIDS. Clin Infect Dis 1993; 17: 837-42.

27.- Centers for Disease Control and Prevention. Update: trends in AIDS incidence, deaths and prevalence-United States, 1996. MMWR Morb Mortal Wkly Rep 1997; 46: 165-73.

28.- van der Horst C M, Saag M S, Cloud G A, Hamill R J, Graybill J R, Sobel J D, et al. Treatment of cryptococcal meningitis associated with the acquired immunodeficiency syndrome. N Engl J Med 1997; 337: 15-21.

29.- Brouwer A E, Rajanuwong A, Chierakul W, Griffin G E, Larsen R A, White N J, et al. Combination antifungal therapies for HIVassociated cryptococcal meningitis: a randomised trial. Lancet 2004; 363: 1764-7.

30.- Perfect J R, Dismukes W E, Dromer F, Goldman D L, Graybill J R, Hamill R J, et al. Clinical practice guidelines for the management of cryptococcal disease: 2010 . Update by the Infectious Diseases Society of America. Clin Infect Dis 2010; 50: 291-322.

31.- Bozzette S A, Larsen R A, Chiu J, Leal M A, Jacobsen J, Rothman P, et al. A placebocontrolled trial of maintenance therapy with fluconazole after treatment of cryptococcal meningitis in the acquired immunodeficiency syndrome. California Collaborative Treatment Group. N Engl J Med 1991; 324: 580-4. 